Escarbajal-Frutos, A. (2014). La educación intercultural en los centros educativos. Revista Electrónica Interuniversitaria de Formación del Profesorado, 17 (2), 29-43.

DOI: http://dx.doi.org/10.6018/reifop.17.2.197291

\title{
La educación intercultural en los centros educativos
}

\author{
Andrés Escarbajal Frutos \\ Universidad de Murcia
}

\section{Resumen}

En el presenta artículo se analiza la educación intercultural en los centros educativos como factor determinante en la construcción de sociedades democráticas e inclusivas y también como apuesta por la eficacia, la equidad y la calidad como elementos inseparables del sistema educativo. Se hace una contextualización del momento actual y del papel que juegan y deben jugar los centros educativos para responder a los retos planteados por la creciente presencia de alumnos extranjeros, una vez comprobado que la segregación en las aulas puede determinar igualmente la marginación social en unos centros educativos diseñados para la homogeneización y estructurados en base a una lógica del control. Del mismo modo, en el artículo se expone que poco puede hacer un centro educativo para fomentar la igualdad la equidad y la calidad si las políticas económicas y sociales no caminan por el mismo sendero. Finalmente, se dan algunas claves de reflexión para apostar por metodologías que tengan en cuenta que una mente intercultural vitaliza el pensamiento de los alumnos, refuerza la solidaridad y el trabajo cooperativo y es la base para la construcción de entornos democráticos.

\section{Palabras clave}

Inclusión; Educación Interculturalidad; Calidad; Equidad.

\section{The Intercultural Education in schools}

Contacto

Andrés Escarbajal Frutos, andreses@um.es, Departamento de Didáctica y Organización Escolar. Facultad de Educación. Campus de Espinardo. 30100. Murcia. España. 


\begin{abstract}
In the present article analyses intercultural education in schools as a factor in building democratic and inclusive societies as well as commitment to efficiency, equity and quality as integral parts of the education system. Contextualization of the moment and of the role is done and should do the educational centers to respond to the challenges posed by the growing presence of foreign students after verification that segregation in the classroom can also determine the social marginalization in the centers educational designed for the homogenization and structured based on a control logic. Similarly, the article states that little a school can do to promote equality and equity if the economic and social policies do not walk the same path. Finally, given some key reflections to bet on methodologies that take into account intercultural mind that vitalizes the students' thinking, strengthens solidarity and teamwork and is the basis for building democratic environments
\end{abstract}

\title{
Keywords
}

Inclusion; Intercultural education; Quality; Equity.

\section{Introducción}

El tercer milenio se inició con la creciente interdependencia mundial tanto en lo económico como en los ámbitos científico, cultural y político, en un planeta cada vez más pluricultural; aunque quizá no seamos demasiado conscientes de que esta época en la que nos ha tocado vivir está produciendo cambios radicales a un ritmo vertiginoso, capaces de replantear la relación del hombre (en términos antropológicos) con el mundo, con la cultura, con los demás y consigo mismo. Entre otras consecuencias, para la educación estos cambios reclaman un giro copernicano en sus planteamientos para dar respuesta a las diferencias culturales, pero también lingüísticas, políticas, religiosas..., de un mundo interconectado por múltiples vías (Cambi, 2010; Rovatti, 2008). Ante esta situación, la diversidad cultural debe ser el motivo que nos conduzca no sólo al conocimiento del otro basado en descripciones etnográficas, sino al descubrimiento del otro como sujeto singular y universal a la vez. El otro, desde posiciones éticas, no debe ser visto desde su identidad, menos aún desde sus diferencias con nosotros, sino desde el respeto a su complejidad y sus características de todo tipo.

En este sentido, es conocido que los procesos migratorios, entre otras consecuencias, generaron la necesidad de investigar pedagógicamente para dar respuesta, desde el trabajo educativo, a la salvaguarda del individuo como ser social comunitario. En efecto, se trataba de dar nuevo sentido a las motivaciones, la naturaleza y la dirección de los discursos pedagógicos desde una perspectiva intercultural. Sin embargo, el debate sobre la interculturalidad y la educación intercultural no es tanto una obligación pedagógica (que también lo es) como una necesidad; una necesidad de revisar la propia epistemología de la que hemos estado bebiendo (Pizzi, 2008).

Hoy, cuando parecía que la economía y la capacidad emprendedora eran la opción sobre la que debían girar las prácticas educativas, vuelve a surgir el pilar de Aprender a convivir convirtiéndose en una prioridad básica (Gómez, 2012), aprender a reivindicar de nuevo la diferencia como valor, no como riesgo, como sinergia integradora (en el mejor de los sentidos) y compleja. Y eficaz, porque de eso también se trata en educación, de conseguir los objetivos de optimización propuestos, aunque sabemos que en educación el término eficacia no tiene las mismas connotaciones que en otros ámbitos sociales. Pero también 
somos conscientes que frente al racismo, la xenofobia, los prejuicios, la marginación y exclusión..., la educación debe actuar de forma constante y, si se permite la expresión, radicalmente, desde el punto de vista práctico, pero también del teórico (Cambi, 2010). El discurso educativo se tiene que analizar como elemento dependiente e íntimamente relacionado con los restantes elementos de la estructura social.

En ese contexto, los centros educativos no pueden (ni deben) sustraerse, pues se ven profundamente afectados por lo que Habermas (2000) llamó "la explosión de la globalización y la multiculturalidad" (p. 90). Conocemos el poder de transformación que tienen las instituciones educativas y su enfoque restaurativo (Roldán y Cantillo, 2012), siendo las prácticas más comunes la mediación entre iguales y las estrategias didácticas grupales para favorecer la interculturalidad, mejorar el clima de aula, la convivencia, el respeto y el diálogo. Esas prácticas en los centros educativos pueden generan inseguridad, ansiedad e incertidumbre en los docentes, porque la sociedad sigue manteniendo la firme convicción de que muchos de los problemas sociales actuales podrían ser solucionados si sabemos cómo educar desde las instituciones educativas (Portera, 2006), y ello hace que se ejerza cierta presión en los docentes.

Esa presión sobre los docentes se agudiza porque también se ha demostrado (Leiva, 2008) que la configuración y organización del sistema escolar español es poco propicia para desarrollar la educación intercultural. Por eso se hace necesario un replanteamiento de todo el sistema educativo, al menos, en tres direcciones (Ytarte, en FETE-UGT, 2010):

a) Desarrollar un proceso cultural de reconocimiento normalizado de la diversidad y de sus narrativas plurales en todas sus manifestaciones: revisar y modificar los currículos educativos en los que se trata la diversidad como diferencia; poner en valor y promocionar las producciones culturales de todos los alumnos, sin etiquetar ni clasificar; y promocionar el multilingüismo.

b) Implementar un proceso social de elaboración y compromiso con los valores y principios democráticos.

c) Completar lo anterior con un proceso político e institucional orientado a articular programas socioeducativos que favorezcan un marco común de convivencia y sentido de pertenencia a una comunidad: desarrollo de pactos de convivencia y civismo en y con las comunidades; favorecer la transversalidad en proyectos de interés común; y poner en marcha los planes globales de acción comunitaria.

En cualquier caso, debemos reconocer que, a pesar de los miles de artículos y libros sobre interculturalidad, quizá todavía hoy nos falte aplicar el concepto gramsciano de praxis, integrar en una misma acción teoría y práctica o, como escribe Zapata-Barrero (2013), "mostrar que existe una teoría detrás de una práctica intercultural..., pasar de un período de retórica de la interculturalidad a otro más de acción" (p. 5).

\section{Los centros educativos y la inclusión}

Algunos autores (García y Goenechea, 2009) analizaron la evolución de los modelos de atención a la diversidad en el sistema educativo español desde los años 60/70, basados en principio en la integración y la adaptación curricular, para pasar después, en la década de los 90, a apostar por el modelo inclusivo, basado en la modificación del sistema educativo para responder a todos los alumnos. Y a este respecto, escribieron Núñez y Romero (2008) que la educación debía encontrar sus anclajes, más allá de lo meramente instrumental y tecnológico, en su función social y en su dimensión humana. Y, sostenían si de verdad 
queremos hacer una auténtica revolución en nuestras metodologías educativas, no nos debemos parar en las técnicas, en los instrumentos o en los procesos, sino escrutar en los principios que nos moverán a la acción. Entre esos principios, sin duda uno de los más importantes es el de la inclusión, que proporciona a los alumnos vínculos para construir juntos un tipo de educación que los haga más libres, autónomos y responsables. Es un principio que busca afinidades esenciales para la vida colectiva, teniendo en cuenta la diversidad cultural y presente en sus aulas (Arnaiz y Escarbajal, 2011).

Un centro educativo intercultural, inclusivo, es aquel que tiene una visión nueva de las relaciones entre culturas y es capaz de modificar e incluso transformar la estructura misma de la organización y los métodos de formación, las relaciones entre los docentes, los alumnos, las familias..., pero, sobre todo, la perspectiva con que miran los saberes y las disciplinas (Santerini, 2010). Como escribe Casanova (2011), "si las personas diferentes deben vivir y convivir juntas, lo mejor será que se implemente un sistema educativo en el que se eduquen juntas" (p. 95). Este principio es precisamente la base del modelo inclusivo de educación (Ainscow, 2000). La educación inclusiva, su progresión, es como una escalera cuyos peldaños están integrados por culturas inclusivas, políticas inclusivas, prácticas inclusivas, comportamientos inclusivos... Por tanto, la inclusión impregna todos los ámbitos educativos, tanto teóricos como prácticos (Casanova, 2011; Herrán e Izuzquiza, 2010).

La educación inclusiva, además de implicar la implementación de un currículo democrático, abierto y flexible, con los recursos pertinentes y adecuados y una organización escolar que lo permita y potencie:

Debe constituir un núcleo aglutinador de la sociedad, que colabore activamente en el centro educativo para que éste se convierta en una comunidad de aprendizaje real, en la que todos participen y aporten su riqueza individual y grupal a la mejora de cada uno de sus integrantes. (Casanova, 2011, p. 99)

Pero si los centros educativos deben fomentar el respeto por la diversidad y favorecer la inclusión, también deben tener cuidado de que el énfasis que ponen en ello no desemboque en trato desigual a los diferentes, porque, al fin y al cabo, somos mucho más iguales que distintos (Arroyo, 2013; Carbonell, 2002). Es también importante establecer la visión que tenemos de la inclusión para orientar las acciones y crear un marco conceptual compartido por todas las instituciones. $\mathrm{Y}$, a este respecto existen cuatro elementos que pueden ser de utilidad a aquellos que quieran "examinar su propia definición funcional" ya sean directivos de centros escolares, supervisores o profesores (Echeita y Ainscow, 2011):

- La inclusión es un proceso.

- La inclusión busca la presencia, la participación y el éxito de todos los alumnos.

- La inclusión precisa la identificación y la eliminación de barreras.

- La inclusión pone particular énfasis en aquellos grupos de alumnos que podrían estar en riesgo de marginalización, exclusión o fracaso escolar.

Vivimos en un mundo heterogéneo, diverso, y la educación, los currículum educativos, deben preparar al alumnado para este contexto e identificar las barreras que se oponen al aprendizaje y la participación (García y Goenechea, 2011), pues uno de los objetivos más importantes será ofrecer una educación de calidad, para todos bajo el marco de la educación inclusiva. El currículum intercultural, si de verdad pretende ser inclusivo, debe ser para todos, no sólo para los extranjeros o inmigrantes; cuando hablamos de Planes o Proyectos educativos interculturales hablamos también de una comunidad escolar colaboradora, de cultura escolar inclusiva y de prácticas en las aulas y fuera de ellas 
(Gallego, Rodríguez y Lago, 2012). Todo ello implica la selección, elaboración y desarrollo de un currículum que no silencie las voces de ninguna cultura ni ninguna clase social. Construir un currículum que contribuya al crecimiento mutuo, evite respuestas segregadoras y centradas en el déficit, para garantizar la igualdad, la calidad y la cohesión social (García-Fernández, 2012). Y todo ello pasa, desde nuestro punto de vista, por la educación intercultural.

\section{Los centros educativos y la interculturalidad}

Teniendo en cuenta lo expresado en el punto anterior, y si tenemos el convencimiento de que las diferencias son positivas por cuanto enriquecen la cultura, estaremos igualmente de acuerdo en que los centros educativos deberán "preparar a sus alumnos para vivir y convivir en entornos cambiantes y heterogéneos" (Arroyo, 2013, p. 145). Sin embargo, somos conscientes de lo que escribe Munhoz (2002), que el concepto "diferencia" designa una problematización en la dicotomía normalidad/anormalidad. Los centros educativos, sobre todo la escuela, fueron pensadas para la homogeneización: los espacios, los tiempos, la organización, la clasificación por edades y cursos, los contenidos, los métodos... En este escenario, la diferencia ha sido vista tradicionalmente como amenaza para ese objetivo homogeneizador, ya que pone encima de la mesa denuncias de situaciones y disonancia con las verdades instituidas. Pero también la diversidad cultural es negada en espacios y centros educativos que se consideran homogéneos por "la no presencia" de minorías (Osuna, 2012). Como escribió Besalú (2008) refiriéndose a la escolarización del alumnado extranjero:

... las acciones llevadas a cabo, los planes diseñados, y los materiales y recursos usados responden más a una lógica que pretende defender a la escuela (profesorado y alumnado) de la presunta influencia desestabilizadora del alumnado extranjero, que a la idea, tachada a menudo de retórica y de políticamente correcta es decir, inservible e irreal- de, por ejemplo, ofrecer, también al alumnado extranjero, una educación de calidad, o de revisar la organización y el funcionamiento de la escuela para hacerla mejor para todos, más inclusiva y más eficaz. (p. 87)

Esta situación se da igualmente en las relaciones educativas basadas en la lógica del control. Por eso, el problema que se plantea en los centros educativos con la diversidad cultural no puede ser resuelto desde posicionamientos instrumentales, sino que es necesaria una respuesta ética que tenga en cuenta la riqueza de la diversidad cultural (Abdallah-Pretceille, 2006). Pero respuesta ética no quiere decir asumir un listado de valores que todos deban respetar, sino una construcción de normas, estilos de comportamiento y, por supuesto valores, asumidos democráticamente y basados en el respeto de todos los alumnos, sean cuales fuesen sus circunstancias. Y esta es precisamente la verdadera dificultad para conseguir una educación intercultural: buscar el acuerdo ético de la diversidad; una premisa básica e ineludible antes de emprender acciones educativas desde cualquier metodología didáctica.

Pero el problema no está en la diversidad en sí, sino en la situación de segregación a que se ven sometidos los "diferentes", la discriminación que perciben y la ausencia de políticas públicas que promuevan el conocimiento y la interacción entre culturas en un marco de ciudadanía común (Zubero, 2010). Y, en este sentido, el sistema educativo español, contiene importantes elementos de discriminación por cuanto "favorece a los favorecidos y ofrece escasas posibilidades a los desfavorecidos, que son habitualmente los que experimentan el fracaso escolar" (Mayoral, 2010, p. 2). Así, una investigación (Martínez, 
2011) analizó el fracaso escolar como una forma de exclusión del derecho a la educación, demostrando que las medidas extremas de atención a la diversidad que se han ido desarrollando en la ESO promueven vías paralelas y devaluadas de escolarización y, por consiguiente, nuevos senderos para la exclusión.

Por ello, si el sistema educativo segrega a ciertos grupos de alumnos en aulas, programas o modelos diferentes por su pertenencia a determinados grupos de referencia (nacionalidad, lengua, religión, clase social, etc.) o porque no tienen "base suficiente", no tienen "competencias instrumentales" o no han adquirido "niveles mínimos", se corre el riesgo de penalizar, de estigmatizar al estudiante antes incluso de ofrecerle la oportunidad de aprender (Aguado, 2009). Y sostiene esta autora que apostar por la interculturalidad supone el compromiso de abandonar visiones restrictivas que la asocian a grupos sociales específicos (inmigrantes, gitanos, marginales) y asumirlo como enfoque que se dirige a todos y cada uno de los miembros de la sociedad y que afecta a todas las decisiones que se adoptan en un centro educativo.

Pero las políticas de excelencia educativa, tal como las entiende la Unión Europea, no son políticas de equidad, porque no apuestan por la inclusión como concepto y praxis pedagógica, por la democracia y por el ejercicio de la ciudadanía (Besalú, 2011). De este modo, la propia OCDE, en su informe Equidad y calidad de la educación. Apoyo a estudiantes y escuelas en desventaja (2012) advierte de que la separación temprana de alumnos, la segregación, eleva el fracaso escolar. Además, remarca que los alumnos procedentes de entornos socioeconómicos desfavorecidos tienen dos veces más de probabilidades de caer en el fracaso escolar y en el abandono de las aulas. Estas conclusiones son muy significativas si las valoramos teniendo en cuenta que el Informe PISA decía en 2009 que, pese a los mediocres resultados de los alumnos españoles, el análisis de resultados resaltaba que España tenía un sistema educativo muy equitativo, que integraba bien a los alumnos de entornos socioeconómicamente desfavorables. Entonces, ¿qué ocurre?, ¿Son incompatibles los éxitos escolares con la equidad en educación?, ¿Son irreconciliables calidad y equidad? Sea cual fuere la respuesta, desde luego para nosotros no son términos incompatibles, y lo que nos gustaría destacar es que la segregación no es una opción ni para la equidad ni para la calidad.

Precisamente, para intentar dar respuesta a ese tenor, estamos realizando una investigación, en una zona concreta de la Región de Murcia, sobre el éxito educativo del alumnado inmigrante, una zona que fue receptora de fuerte inmigración, que después cayó en la marginación de los colectivos inmigrantes por la crisis económica, y que ahora intenta recuperar el pulso del desarrollo. Concretamente, estamos analizando las competencias matemáticas y lingüísticas de los alumnos inmigrantes de $4^{\circ}$ de la Educación Secundaria Obligatoria en los Institutos de esa zona para comprobar después el número de alumnos extranjeros que finalmente obtienen el título de la ESO sin recibir ninguna adaptación ni medida específica de atención a la diversidad.

Los Institutos de Educación Secundaria analizados tienen más de un 30\% de alumnos inmigrantes matriculados en $4^{\circ}$ de la ESO, de los cuales un $28 \%$ son ecuatorianos, un $17 \%$ colombianos, un $12 \%$ marroquíes, un $10 \%$ rumanos y un $5 \%$ ucranianos; con presencia también de alumnos procedentes de Bolivia, Brasil, Uruguay, República Dominicana, Perú, Georgia, Paraguay, Bulgaria y China. Los primeros datos nos avanzan que un 29 '4\% de los alumnos inmigrantes no obtienen el título de la ESO, siendo en su mayoría chicas procedentes de Ecuador y Colombia; y un 70'5\% de los alumnos inmigrantes sí obtienen el título de la ESO.

Nuestro propósito es ampliar la muestra a toda la Región de Murcia, así como introducir otras variables en la investigación, tales como analizar la metodología utilizada en el aula, el 
nivel socioeconómico de las familias de estos alumnos, los recursos culturales existentes en el hogar, y el uso de la lengua materna en casa como factores influyentes en el rendimiento académico y en la calidad y equidad educativas.

Por otra parte, que los alumnos adquieran la competencia intercultural implica interactuar de forma efectiva y apropiada con personas de otras culturas, y ello, a su vez, implica atender a los conocimientos, actitudes, habilidades y comportamientos (Perry \& Southwell, 2011); exige conocimientos, motivación, habilidades verbales, comunicación no verbal y comportamientos adecuados y eficaces (Lustig \& Koester, 2006); tolerancia a la ambigüedad de comportamiento, flexibilidad, consciencia comunicativa, descubrimiento del otro, empatía (Hiller \& Wozniak, 2009). Así, la competencia intercultural se convierte en "un conjunto de habilidades cognitivas y características afectivas y de comportamiento que hacen desarrollar una efectiva interacción en una variedad de contextos culturales" (Bennett, 2008, p. 16).

Una mente monocultural va perdiendo paulatinamente la capacidad para la innovación, la curiosidad por explorar nuevos horizontes, la reflexión sobre su propia naturaleza, y se cierra sobre sí misma a través de repeticiones compulsivas. Por el contrario, la mente intercultural vitaliza el pensamiento creando la necesidad de buscar continuamente nuevos horizontes, nuevos estímulos, originales saberes, sin los cuales la creatividad se quedaría fosilizada. El alumno que aprende interculturalmente se sorprende, se muestra curioso, explora, investiga, inventa... (Demetrio y Favaro, 2009).

Desde la perspectiva de la inclusión de los alumnos extranjeros, la educación intercultural garantiza la igualdad de oportunidades formativas. Este debe ser el primer objetivo, irrenunciable, de una institución educativa democrática. Pero poco puede hacer un centro educativo para conseguirlo si las políticas sociales y económicas no marchan por el mismo camino, porque hay estudios que demuestran las dificultades de los inmigrantes para acceder a puestos de trabajo en iguales condiciones que los autóctonos, a pesar de tener la misma titulación (Catarsi, 2010).

Por tanto, el papel de los centros educativos, es muy relevante para trazar nuevos caminos, en la medida de sus competencias y posibilidades, porque marcarán los fundamentos de la nueva sociedad intercultural. En efecto, el ámbito educativo posibilita el encuentro privilegiado para promover la ruptura con estereotipos inhabilitantes y también para alcanzar la construcción de identidades compartidas y equilibradas, que permitan profundizar en el objetivo de la integración social. Aunque, naturalmente, estas posibilidades serán más fácilmente realizables si, como se ha señalado anteriormente, hay una seria voluntad en la orientación política para lograrlo, es decir, para incidir en la transformación de los centros educativos y la sociedad para convertirlos en espacios de auténtica relación intercultural democrática.

Un centro educativo intercultural debe contar con un proyecto integral de actuación que contemple todos los ámbitos educativos, éticos y sociales (Gómez, 2010). Este proyecto debe hacer énfasis en el esfuerzo por contrarrestar cualquier forma de exclusión, promover y fomentar valores y actitudes interculturales y cambiar todas aquellas estructuras que dificulten la consecuencia de lo anterior. Los centros educativos deben transmitir que no podemos entender el discurso de la interculturalidad sin unos esquemas cognitivos y afectivos conectados entre sí; como también es insuficiente conocer las diversas culturas para una comprensión intercultural (Hill, 2006). Por eso, aumentar la sensibilidad intercultural (Straffon, 2003) conduce a aumentar la competencia intercultural, que incluye comunicación y actitudes (Perry \& Southwell, 2011). 


\section{Algunas reflexiones y pautas para la acción}

Hace ya algunos años, Banks (2002) identificó cinco dimensiones de la educación multicultural: la integración de contenidos, la construcción de conocimiento, reducir la discriminación, pedagogía de la equidad, empoderamiento de la cultura y estructura social de la escuela, que todavía hoy no se está llevando a cabo en su totalidad en muchos sistemas educativos europeos y americanos. Quizá por eso, diversos autores (Heilman, 2012; Keddie, 2012; May y Sleeter, 2012) han criticado la incapacidad que tiene la educación multicultural liberal para hacer visible y enfrentarse de forma seria a la injusticia sociales y culturales.

Y es que la diversidad cultural de nuestra sociedad requiere una amplia reflexión sobre el rol de los centros educativos en la formación de ciudadanos, en la manera de educar para la convivencia en la diversidad (no sólo cultural), y en el conocimiento de los derechos y deberes de cada uno (Santerini, 2010). Así, el Consejo de Europa, en el Libro Blanco sobre el diálogo intercultural (2008) ya declaraba que la educación intercultural debería tener en cuenta la pluralidad y la diversidad lingüística y sociocultural a todos los niveles: organizativo, contenidos, programas y métodos... En todas las asignaturas se debía introducir el hecho pluricultural y analizarlo de manera crítica, independientemente de la presencia física de alumnos de varias culturas.

Hoy se habla ya de una educación intercultural de "segunda generación", en la que la enseñanza debería renunciar a las visiones monoculturales y nacionales para adoptar una óptica más amplia y compleja de la humanidad. Por eso, seguir desarrollando modelos educativos monoculturales cuando la sociedad no es homogénea es como considerar que el éxito de los alumnos inmigrantes reside exclusivamente en si ha adquirido la competencia lingüística (García Castaño y Rubio, 2011). La educación intercultural de "segunda generación" se inserta en una sociedad donde la diversidad es la norma y afronta la inmigración como un hecho nada transitorio, sino estable y primordial para construir un futuro de convivencia. Por tanto, ya no se trata como en los objetivos interculturales de "primera generación" de que los centros educativos ayuden a los hijos de inmigrantes a integrarse en el sistema social de acogida, sino de redefinir el principio de igualdad de oportunidades para todos los alumnos, sea cual sea su origen cultural y social (Santerini, 2010).

Muchos de los contenidos y competencias que los centros educativos enseñan quedan pronto obsoletos por los rápidos cambios sociales que estamos experimentando. Esto lleva, otra vez, a plantear la cuestión de qué se debe enseñar: ¿conocimientos o estrategias para acceder a ellos de manera autónoma y permanente?, ¿enseñar o aprender a aprender, adquirir competencias y conocimientos generales básicos y flexibles que puedan ayudar a la formación científica?. Y, sobre todo, ¿deben centrase las instituciones educativas en el alumno o plegarse a la exigencias del mercado? Finalmente, a la hora de formar, ¿se debe pensar en el presente o en el futuro? (Fiorin,. 2010). No tenemos respuesta para estas preguntas, pero sí estamos convencidos de que un programa de educación intercultural debe evitar la introducción fragmentada de contenidos de las culturas minoritarias en el currículo, así como la folclorización de las culturas (García, García y Moreno, 2012). Debe contribuir a potenciar la reflexión social y la autocrítica. La interculturalidad debe ayudar a comprender la realidad desde distintos puntos de vista, desarrollar una visión plural del mundo, evitar el etnocentrismo y mejorar el rendimiento escolar.

Sin embargo, no es una novedad que los centros educativos actúan como filtro que selecciona y determina la clase y cantidad de conocimientos y habilidades ofrecidos a los alumnos, además de evaluar los niveles de adquisición del conocimiento y establecer las 
calificaciones que permitan el acceso a la educación superior, empleo y formación profesional. Como esto es así, en una sociedad pluricultural como la nuestra se hace ineludible ofrecer igualdad de oportunidades, "asegurar que todos los grupos sociales y culturales accedan y tengan un sistema escolar justo y efectivo para todos" (Aguado, 2009, p. 175). Las culturas, las políticas y las prácticas educativas presentes en los centros educativos deben sustentarse en los principios de la interculturalidad y la inclusión. Trazar una educación para todos significa acoger los fines planteados por el movimiento de la educación inclusiva e intercultural y ofrecer respuestas acordes a dichos fines. La comunidad educativa debe conocer en profundidad los significados e implicaciones del tipo de educación a desarrollar; y conjuntamente, docentes, familias e instituciones deben trazar el proyecto educativo del centro así como los procesos y las estrategias a poner en marcha para alcanzar dichos propósitos. La enseñanza es un proyecto vital que necesariamente pasa por las instituciones educativas, pero es un error pensar que se agota en ellas.

Tampoco los centros educativos aseguran el tradicional papel de agencias socializadoras. Antes los marcos de referencia normativos, las visiones del mundo y de la propia identidad eran provistos por la escuela, la familia y la Iglesia, que garantizaban la cohesión social, pero ahora el individuo está aislado frete a una colectividad cada vez más anónima. La inclusión social parece más una cuestión individual que colectiva. A la educación le cabe entonces formar el sentido de pertenencia, desarrollar la capacidad para articular identidades complejas, con pertenencias a múltiples ámbitos. $\mathrm{Y}$, en este sentido, sigue siendo fundamental el papel de los centros educativos para desarrollar el sentido plural de pertenencia, lo cual significa combinar la adhesión y la solidaridad local con la apertura a las diferencias; ello poniendo en práctica dispositivos educativos que fomenten la construcción de experiencias democráticas, Pero, respetando la autonomía de cada centro, una institución educativa no puede operar al margen de otras instancias sociales, sino actuar como catalizadora en la construcción de redes sociales comunitarias que la saquen de su tradicional aislamiento (Tedesco, 2000).

Lo anterior significa que el primer paso será conocer el sentido de la educación intercultural e inclusiva, a qué compromete comunitariamente y realizar una firme apuesta por la misma (De Haro, 2009). Después se verá el cómo; pero desde luego la aproximación intercultural no debería traducirse en intervenciones de tipo "sumativo" (en las clases programadas de antemano se añaden contenidos inherentes a los alumnos hijos de inmigrantes presentes en el aula), ni siquiera solamente en la realización de acciones ad hoc a poner en práctica en añadidura o en sustitución de los programas previstos (como proyectos o actividades circunscritas: por ejemplo, la "semana intercultural", "la hora de la interculturalidad").

Tampoco se trata de elaborar un modelo didáctico específico intercultural, sino que es preciso incluir la perspectiva intercultural en cada disciplina impartida y en cada actividad. Más aún, se trata de que los propios centros educativos sean realmente interculturales en todos los sentidos, implicando naturalmente a toda la comunidad (Escarbajal, 2009). En este sentido, Soto (2006) plantea tres axiomas para afrontar la experiencia y el trabajo intercultural en el aula:

a) Considerar la diversidad cultural de todas y cada una de las personas implicadas en el proceso educativo.

b) Extremar la prudencia ante las informaciones sobre culturas de origen del alumnado y sus familias.

c) Dotar de protagonismo la experiencia concreta, particular e individual y repensar las perspectivas abstractas, globales y colectivas. 
En todo caso, y como señala Nuzzaci (2007), tres son las condiciones indispensables para conseguir aprendizajes interculturales:

a) La capacidad de comprender al otro sin negar la diferencia y sin que deje de ser él mismo.

b) La capacidad de trabajar sobre las divergencias y conflictos, los malentendidos, las emociones y los sentimientos que suscitan las diferencias de valores, estilos de vida y comportamientos.

c) La capacidad y la voluntad para cooperar en la construcción de objetivos comunes.

Además, para profundizar en el discurso intercultural en el que la cultura no sea vista como un repertorio de normas, usos, tradiciones, etc., sino como modalidad de relación y de interacción con el contexto, la dinámica de la clase debe estar orientada hacia la construcción de un saber estar con el otro que consolide "una ética del respeto y de la solidaridad como conocimiento de la interacción y como fundamento de las relaciones" (Grange, 2007, p. 42). Como escribe Naïr (2005):

Cada uno tiene derecho a aceptar o rechazar los valores y creencias del otro. Pero eso no debe impedir el reconocimiento del derecho de cada uno a creer y pensar en función de sus propios valores. Educar en la diversidad es aceptar el encuentro, aprender el lenguaje del otro, correr el riesgo de la confrontación, juzgar al otro no en relación con yo mismo, sino en relación con sí mismo. (p. 4)

Por eso, ante la pregunta: ¿Cómo podemos desde los centros educativos ayudar a prevenir los estereotipos y prejuicios sobre los alumnos hijos de inmigrantes?, la respuesta tiene que ver con la didáctica que emplearemos, pero también con la inteligencia emocional de los alumnos. Debemos reconocer las historias personales, las aportaciones de todas las culturas, sus formas de comunicación, cómo organizan sus espacios vitales..., todo ello con el concurso de mediadores interculturales y evitando siempre las etiquetas. Pero, sobre todo, cuando se tienen objetivos comunes y se trabaja en grupo para conseguirlos se refuerzan los alzos afectivos que evitan las descalificaciones en virtud del color de la piel o la procedencia (Milan, 2007).

$\mathrm{Y}$, en ese sentido, si uno de los objetivos de la educación intercultural es propiciar la colaboración, la solidaridad y la convivencia entre los alumnos, las políticas y prácticas educativas que fomentan la segregación no pueden ser llamadas interculturales (Osuna, 2012). También Rodríguez (2009) demostró la ineficacia de la educación compensatoria para los alumnos extranjeros. Cuando se clasifica a los alumnos se tiende a poner a los hijos de inmigrantes en clases compensatorias, lo cual refuerza las disparidades académicas y la alienación social. Por ejemplo, la medida de acoger al alumno e introducirlo en la lengua del país receptor es incompleta si no va acompañada de otras medidas relacionadas con los compañeros, las asignaturas, la organización del centro, el trabajo con las familias..., pero, en cualquier caso, debe ser una medida coyuntural que ni segregue a los recién llegados ni se extienda mucho en el tiempo (Santerini, 2010). No debe ser tenida, por tanto, como un objetivo en sí mismo.

Además, Flecha, en una de las propuestas (la 2) recogidas en el Libro Blanco de la Educación Intercultural (FETE-UGT, 2010), nos recuerda que hace décadas que las investigaciones internacionales en ciencias sociales y de la educación han demostrado que tanto el tracking (separación de alumnos por habilidades en diferentes centros) como el streaming (separación de alumnos por habilidades en el mismo centro educativo) genera y reproduce desigualdades. Por eso, en su propuesta aboga por las agrupaciones heterogéneas, por ser las que la experiencia ha demostrado que generan mejores rendimientos en todos los 
sentidos. En este agrupamiento mixture no "se saca" a nadie del aula ni se discrimina a nadie. Son grupos interactivos basados en el aprendizaje dialógico, en donde cada alumno es, a la vez, sujeto que aprende y recurso de aprendizaje para los compañeros. Por eso apostamos por un trabajo en grupo cualitativo en el que los alumnos participen en el desarrollo de estrategias de aprendizaje, en los objetivos y metas, al mismo tiempo que se responsabilicen de qué aprender y cómo aprender (Zoletto, 2007).

Teniendo en cuenta todo lo anterior, nos parece interesante destacar la propuesta de García Fernández (2010): buscar enfoques afectivo-sociales para que todos los alumnos vivencien situaciones sociales y personales de comunicación intercultural; educar en valores que desarrollen la autonomía moral de los alumnos y el juicio crítico; ello mediante actividades en las que se pongan encima de la mesa casos de dilemas morales, debates, asambleas, etc; utilizar los enfoques cooperativos, basados en la interdependencia, la responsabilidad y la ayuda mutua; hacer una exposición crítica de la realidad que sea comprensible por todos los alumnos, para que puedan así actuar responsablemente en la sociedad de manera transformadora; importante es también para un proyecto educativo intercultural la gestión democrática del centro, con la participación de las familias, una organización flexible de los espacios, el trabajo en equipo del profesorado, con elaboración propia de materiales didácticos y, cómo no, plantear el propio barrio y la escuela como recursos educativos.

\section{Bibliografía}

Abdallah-Pretceille, M. (2006). Tra etiche e pragmatismo nell'educazione interculturale. In A. Portera (Ed.), Educazione interculturale nel contesto internazionale (pp. 110-116). Milano: Angelo Guerini.

Aguado, T. (2009). Pedagogía intercultural. En A. García y A. Escarbajal de Haro (Eds.), Pluralismo sociocultural, educación e interculturalidad (pp. 167-182). Badajoz: Abecedario.

Ainscow, M. (2000). The next step for Special Education. 5es. Jornades Tècniques d'Educació Especial. Barcelona: APPS.

Arnaiz, P., y Escarbajal Frutos, A. (2011). La inclusión del alumnado extranjero. Prácticas en Educación Intercultural, 3, 21-34.

Arroyo, M. J. (2013). La educación intercultural: un camino hacia la inclusión educativa. Inclusive Education Journal, 6 (2), 144-159.

Banks, J.A. (2002). An Introduction to Multicultural Education. Boston: Pearson.

Bennett, J.M. (2008). On becoming a global soul. In V. Savicki (Ed.), Developing intercultural competence and transformation: Theory, research and application in international education, (pp.13-31). Sterling VA: Stylus.

Besalú, X. (2008). ¿Cómo "defenderse” del alumnado extranjero? Cuadernos de Pedagogía, $380,86-89$.

Besalú, X. (2011). Interculturalidad, todavía. T. E., 319, 12-13.

Cambi, F. (2010). Cittadinanza e globalizzazione. Una sfida educativa e pedagogica. In A. Portera, P. Dusi \& B. Guidetti (Ed.), L'educazione interculturale alla cittadinanza. La scuola come laboratorio. (pp. 29-34). Roma: Carocci. 
Carbonell, F. (2002). Desigualdad social, diversidad cultural y educación. En E. Aja et al., La inmigración extranjera en España. Los retos educativos (pp. 99-118). Barcelona: Fundación la Caixa.

Casanova, M. A. (2011). Educación intercultural: de la política al aula. En E. Soriano, (Ed.), El valor de la educación en un mundo globalizado (pp. 93-135). Madrid: La Muralla.

Catarsi, M. (2010). Per una scuola aperta a tutti. Immigrazione e educazione interculturale. In R. Roig \& M. Fiorucci (Eds.), Strumenti di recerca per l'innovazione e la qualitá in ambito educativo (pp. 111-120). Roma: Universitá degli Studi.

Consejo de Europa (2008). Libro Blanco sobre el diálogo intercultural. Estrasburgo: Servicio de Publicaciones del Consejo.

De Haro; R. (2009). Educación intercultural: posibilidades y dificultades para desarrollar una escuela para todos. En A. García (Ed.), El diálogo intercultural (pp. 219-243). Murcia: Editum.

Demetrio, D. \& Favaro, G. (2009). Didattica interculturale. Milano: F. Angeli.

Echeita, G. y Ainscow, M. (2011). La educación inclusiva como derecho. Marco de referencia y pautas de acción para el desarrollo de una revolución pendiente. Tejuelo, 12, 26-46.

Escarbajal Frutos, A. (2009). Educadores, trabajadores sociales e interculturalidad. Madrid: Dykinson.

European Commission (2008). Education and migration. Strategies for integrating migrant children in European schools and societies. Bruselas: European Commission.

FETE-UGT (2010). Libro Blanco de la educación intercultural: Madrid: Servicio de Publicaciones de FETE-UGT.

Fiorin, I. (2010). Educazione interculturale alla cittadanza: il ruolo della scuola. In A. Portera, P. Dusi \& B. Guidetti (Eds.), L'educazione interculturale alla cittadinanza. La scuola come laboratorio. (pp. 55-61). Roma: Carocci.

Gallego, B., Rodríguez, H., y Lago, M. (2012). Buenas prácticas en educación intercultural inclusiva. En B. López y M. Tuts (Eds.), Orientaciones para la práctica de la educación intercultural. (pp. 53-68) Madrid: Wolters Kluwer.

García, R., García, J.A., y Moreno, I. (2012). Estrategias de atención a la diversidad cultural en educación. Madrid: Catarata

García, J. A. y Goenechea, C. (2009). Educación intercultural. Análisis de la situación y propuesta de mejora. Bilbao: Wolters Kluwer.

García, J.A. y Goenechea, C. (2011). Identificando buenas prácticas interculturales desde claves inclusivas. Revista Educación y Futuro Digital, 5 (11). Recuperado de http://www.cesdonbosco.com/revista/articulos2011/septiembre11/Mayka.pdf

García Castaño, F. J. y Rubio, M. (2011). ¿Misma cultura, misma religión, misma lengua... y también fracasan? El Ilamado alumnado latinoamericano. En D. Barbolla (Ed.): Migraciones latinoamericanas en la nueva civilización. Conformando identidad, (pp. 279-316). Biblioteca Nueva: Madrid.

García-Fernández, J. A. (2010). Lo organizativo en la escuela intercultural. En AA. VV., Interculturalidad y ciudadanía: red de escuelas interculturales. Recuperado de http://www.escuelas interculturales 
Gómez, J. (2010). Lo ético en la escuela intercultural: proyecto AMANI. En AA. VV., Interculturalidad y ciudadanía: red de escuelas interculturales. Recuperado http://www.escuelas interculturales

Gómez, J. (2012). Convivencia, educación e interculturalidad. En B. López y M. Tuts (Eds.), Orientaciones para la práctica de la educación intercultural. (pp. 125-136) Madrid: Wolters Kluwer.

Grange, T. (2007). La dimensione interculturale nelle pratiche educative. In T. Grange, y A. Nuzzaci, (Eds., Interculturalitá e processi formativi (pp. 35-49). Roma: Armando Edizioni.

Habermas, J. (2000). La constelación postnacional. Ensayos políticos. Barcelona: Paidós.

Heilman, E.E. (2012). Social Studies and Diversity Education. New York: Routledge.

Herrán, A. de la e Izuzquiza M. D. (Eds.) (2010). Las claves del éxito. Madrid: Pirámide.

Hill, I. (2006). Student types, school types and their combined influence on the development of intercultural understanding. Journal of Research in International Education, 5 (1), 5-33.

Hiller, G.G. \& Wozniak, M. (2009). Developing an intercultural competence programme at an international cross-border university. Intercultural Education, 20 (4), 113-24

Instituto de Evaluación (2009). PISA 2009. Programa para la evaluación Internacional de los alumnos. Informe español. Madrid: Ministerio de Educación

Keddie, A. (2012). Educating for Diversity and Social Justice. Nueva York: Routledge.

Leiva, J. (2008). Interculturalidad, gestión de la convivencia y diversidad cultural en la escuela: un estudio de las actitudes del profesorado. Revista Iberoamericana de Educación, 46, 1-14.

Lustig, M.W. \& Koester, J. (2006). Intercultural competence: interpersonal communication across culture. 5 th ed. Boston MA: Pearson.

Martínez, B. (2011). Luces y sombras de las medidas de atención a la diversidad en el camino de la inclusión educativa. Revista Interuniversitaria de Formación del profesorado, 70 $(25,1), 165-183$.

May, S. y Sleeter, C.E. (2012). Critical Multiculturalism, Theory and praxis. Londres: Routledge.

Mayoral, V. (2010). La apuesta por una educación intercultural. En AA. VV., Interculturalidad y ciudadanía: red de escuelas interculturales. Recuperado de http://www.escuelas interculturales

Milan, G. (2007). Comprendere e costruire l'interculturalitá. Lecce: Pensa Multimedia.

Munhoz, A. V. (2002). La diferencia en la institución escolar: las formas jerarquizadas de normalización. Recuperado de http:// educación-critica.blogspot.com

Naïr, S. (2005). Alianza de civilizaciones y diálogo para la modernidad. El País. 12 de marzo, 4.

Núñez, L. y Romero, C. (2008). Pensar la educación. Madrid: Pirámide.

Nuzzaci, A. (2007). Interculturalitá e competemnze nelle "scuole di frontiera", ovvero per un'educazione alla mondialitá. In T. Grange, y A. Nuzzaci, (Eds.), Interculturalitá e processi formativi (pp. 73-101). Roma: Armando Edizioni. 
OCDE, (2012). Informe sobre Equidad y calidad de la educación. Apoyo a estudiantes y escuelas en desventaja. París: OECD Publishinng.

Osuna, C. (2012). En torno a la educación intercultural. Una revisión crítica. Revista de Educación, 358, 38-58.

Perry, L.B. and Southwell, L. (2011). Developing intercultural understanding and skill: models and approaches. Intercultural Education, 22 (6), 453-466.

Pizzi, F. (2008). La pedagogía interculturale in Italia: questioni epistemologiche. Profesorado. Revista de Currículo y Formación del Profesorado, 12 (2), 1-13.

Portera, A. (2006). Educazione interculturale nel contesto internazionale. Milano: Angelo Guerini.

Rodríguez, R. M. (2009). La investigación sobre la educación intercultural en España. Archivos Analíticos de Políticas educativas, 17, (4), 1-29.

Roldán, C. y Cantillo, L. (2012). Formas alternativas a la resolución tradicional de conflictos: Las prácticas restaurativas. En B. López y M. Tuts (Eds.). Orientaciones para la práctica de la educación intercultural (pp. 137-148). Madrid: Wolters Kluwer.

Rovatti, A. (2008). Possiamo addomesticare l'altro? Udine: Forum.

Santerini, M. (2010). Cittadinanza e intercultural nella scuola. Introduzione generale e riflessione sui risultati del gruppo di recerca di Milano. In A. Portera, P. Dusi \& B. Guidetti (Eds.), L'educazione interculturale alla cittadinanza. La scuola come laboratorio. (pp. 65-72). Roma: Carocci.

Soto, P. (2006). Docentes, sujetos culturales y mediadores interculturales. En P. Álvarez, P. et al., Hacia el aula intercultural. Experiencias y referentes (pp. 9-39). Madrid: MEC.

Straffon, D.A. (2003). Assessing the intercultural sesitivity of high school students attending an international school. International Journal of Intercultural Relations, 27 (4), 487501.

Tedesco, J. C. (2000). Educar en la sociedad del conocimiento. Buenos Aires: F.C.E.

Zapata-Barrero, R. (2013). Del diálogo intercultural a la acción intercultural. En R. ZapataBarrero y G. Pinyol (Eds.), Manual para el diseño de políticas interculturales (pp. 5-12). Barcelona: Universidad Pompeu Fabra.

Zoletto, D. (2007). Straniero in classe. Una pedagogía dell'ospitalitá. Milano: Raffaello Cortina.

Zubero, I. (2010). Confianza ciudadana y capital social en sociedades multiculturales. San Sebastián: Ikuspegi. 


\section{Autores}

\section{Andrés Escarbajal Frutos}

Doctor Europeo en Pedagogía Social, Especialista en Mediación Intercultural, Profesor Contratado Doctor Interino de la Facultad de Educación en la Universidad de Murcia, y asesor-consultor de prácticas y políticas educativas. Miembro del grupo de Investigación Educación Inclusiva: Escuela para Todos y, desde hace años trabaja con estrategias de trabajo colaborativo en temáticas relacionadas con la interculturalidad, la mediación y la educación inclusiva. Ha sido Secretario del Centro de Estudios Interculturales de la UMU. Entre sus obras destacan: Educadores, Trabajadores Sociales e Interculturalidad (Madrid: Dykinson); e Interculturalidad, Mediación y Trabajo Colaborativo (Madrid: Narcea). 